\title{
Prevalence of extrapulmonary tuberculosis among people living with HIVIAIDS in sub-Saharan Africa: a systemic review and meta-analysis
}

This article was published in the following Dove Press journal: HIVIAIDS - Research and Palliative Care

\section{Hussen Mohammed' \\ Nega Assefa ${ }^{2}$ \\ Bezatu Mengistie ${ }^{2}$}

'School of Medicine, College of Medicine and Health Sciences, Dire Dawa University, Dire Dawa, Ethiopia; ${ }^{2}$ College of Health and Medical Sciences, Haramaya University, Harar, Ethiopia
Correspondence: Hussen Mohammed School of Medicine, College of Medicine and Health Science, Dire Dawa

University, P.O. Box 1362,

Dire Dawa, Ethiopia

$\mathrm{Tel}+251913416579$

Fax +25। 25 II 2 797।

Email hus.aliya@gmail.com
Background: The double burden of infectious diseases such as tuberculosis (TB) and HIV disproportionately affects Africa. Our objective was to summarize the prevalence of extrapulmonary TB (EPTB) among people living with HIV/AIDS (PLWHA) in sub-Saharan Africa (SSA).

Methods: We searched PubMed and Google Scholar for studies done on the prevalence of EPTB among PLWHA which published in English from 1990 to 2017. We also searched bibliographic indices and browsed through reference lists of articles and journals. We critically appraised the quality of articles and abstracted the data with Joanna Briggs Institute (JBI) checklists. We used Review Manager Software (version 5.3) and Compressive Meta-analysis Software (version 2.2) for analysis. Finally, we summarized the effect estimate by using random-effects model and explored potential sources of heterogeneity by subgroup and sensitivity analyses. We assessed publication bias by funnel plot and statistical test. Finally, we used PRISMA format for reporting. Results: We included, in this review, 31 studies that fulfilled our inclusion criteria, with a total population of 28,659 . The prevalence estimate of EPTB among PLWHA ranged from $6.4 \%$ (95\% CI: $3.8,9.0)$ to $36.8 \%(95 \%$ CI: $28.6,45)$; random-effects pooled prevalence of EPTB among PLWHA was found to be $20 \%$ (95\% CI: 17, 22; heterogeneity: $\tau^{2}=0 ; \chi^{2}=509.09$, degrees of freedom $\left.[d f]=30, P<0.00001 ; P^{2}=94 \%\right)$. No evidence of publication bias was observed $(P=0.44$ for Egger's regression analysis and $P=0.11$ for Begg's rank correlation analysis).

Conclusion: We can conclude from our analyses that the prevalence of EPTB among PLWHA was high. It is necessary to give emphasis for EPTB, especially when screening TB among PLWHA, widening the spectrum of screening to include all EPTB sites in the countries with high EPTB and/or HIV prevalence in the general population.

Keywords: extrapulmonary tuberculosis, HIV, sub-Saharan Africa, systemic review, metaanalysis, co-infection

\section{Introduction}

Tuberculosis (TB) is the ninth leading cause of death worldwide and the leading cause from a single infectious agent that is ranking above HIV/AIDS. The disproportionate burden of HIV and TB infection and disease in Africa at the start of the new millennium continues unabated. In 2016, of all estimated 10.4 million new cases of (25\% in Africa) TB that resulted in 1.7 million (44\% in Africa) deaths worldwide, Africa though, home to only $13 \%$ of the world's population have been living in Africa. Africa bears $30 \%$ of HIV prevalence among TB patients or its co-infection as compared to $1.3 \%$ by the Eastern Mediterranean region. HIV prevalence in TB patients 
or co-infection was low in Eastern Mediterranean region (1.3\%), nevertheless, it was $30 \%$ in Africa. ${ }^{1}$

Furthermore, Africa bears $75 \%$ of about 1.03 million of TB/HIV co-infection cases that occur each year globally. Furthermore, in Africa, TB is often observed first in HIV-infected patients, and it is the leading cause of death among them. ${ }^{1}$ In addition, the UNAIDS global report in 2016 showed that sub-Saharan Africa (SSA) takes the lead by contributing the highest burden of HIV: 70\% (25.5 million) of 36.7 million people living with HIV, $64 \%$ of 1.8 million newly infected, and $73 \%$ of 1.0 million deaths. ${ }^{2}$

Extrapulmonary TB (EPTB) is the TB of organs other than the lungs, eg, pleura, lymph nodes, abdomen, genitourinary tract, skin, joints, bones, and meningeal. ${ }^{3,4}$ A majority of individuals after exposure control the spread of bacillia in pulmonary site, but in others bacilli disseminate through lymphatic or hematogenous system and subsequently affect single or multiple extrapulmonary sites..$^{5}$ The prevalence of EPTB among notified TB cases in African region in 2017 was $16 \%$, which is the second highest next to Eastern Mediterranean region $(24 \%)$, which is more than global prevalence of EPTB (15\%). The lowest prevalence was reported from Western Pacific region $(8 \%) .{ }^{1}$

To mitigate the burden of EPTB/HIV co-infection, the WHO took the initiative. In 2007, it published guidelines on how to diagnose EPTB among HIV-positive people which were based on one culture-positive specimen or histological or strong clinical evidence consistent with active EPTB, followed by a decision by a clinician to treat with a full course of anti-TB treatments. ${ }^{6}$ In addition, the WHO published an up-to-date policy on collaborative TB/HIV activities in $2012 .^{7}$ This policy emphasized the importance of surveillance of HIV among TB patients and vice versa in all countries. Furthermore, in 2013, the WHO updated the policy on the diagnosis of TB using GeneXpert Mycobacterium tuberculosis/rifampicin as an initial diagnostic test at point of care. It could replace microscopy and be used for diagnosis of all presumptive pulmonary TB (PTB) patients, pediatrics patients, TB/HIV co-infected patients, and selected specimen of the diagnosis of EPTB patients. ${ }^{8,9}$

A wide range of estimates for the prevalence of EPTB among people living with HIV/AIDS (PLWHA) have been reported from different SSA countries. For instance, in Ethiopia, more than half, 54.7\% co-infection among HIV/AIDS individuals, had EPTB. ${ }^{10}$ In Cameroon and Ghana, HIV coinfection with EPTB was $37.1 \%$ and $45.2 \%$, respectively. ${ }^{11,12}$ However, an estimate reported on the prevalence of EPTB among PLWHA was inconsistent and unclear.
Therefore, for the dual burden of TB/HIV affecting the Africa, we aimed to summarize and synthesize the prevalence of EPTB among HIV-positive individuals in SSA. This is important for public health policy and planning and development of clinical services that address the needs of EPTB and HIV/AIDS patients.

\section{Methods \\ Study participants}

The study participants included as a sample in reviewed studies could be males and females; all age groups; the time of screening or diagnosing EPTB among HIV had been at the same time (both tested at a time) or after the HIV status is known. The time of reviewed studies performed could be either when HIV/AIDS patients have been in pre-antiretroviral therapy or taking antiretroviral therapy.

\section{Outcome}

This review considered studies that include the prevalence estimate of EPTB among PLWHA.

\section{Eligibility criteria}

For our review, we included the studies that have been done on PLWHA and reported the proportion of co infection for EPTB and HIV. Included studies were observational study design, which includes cohort (prospective or retrospective), cross-sectional or medical review. Studies that were written only in English, health facilities that could be hospitals, health centers or similar that belong to governmental or nongovernmental, or private, and published between January 1, 1990, and November 30, 2017, were included. The screening or investigations were included irrespective of diagnostic methods. Abstracts without full texts, articles without journal name and/or authors, conference proceeding or presentation, studies with total population of less than 50 people, study with incidence, qualitative study, review, case study, TB/HIV co-infection or PTB/HIV only co-infection, and book chapters were excluded from the final analysis.

\section{Search strategy}

A three-stage search strategy was used to identify all relevant published literature. First, an initial search of PubMed database and Google Scholar was made. Second, searching the reference lists or bibliographies of all identified articles was checked manually for articles of interest, and finally gray literature was sought from institutions and government 
websites. Available electronic journal articles were searched through HINARI. We also searched relevant reference lists and relevant journals manually. The dates of publication covered were January 1, 1990, to November 30, 2017. The search engine used with modification was with initial keywords and Medical Subject Headings (MeSH) term for PubMed database: "Tuberculosis, lymph node" OR "tuberculosis, cardiovascular" OR "tuberculosis, central nervous system" OR "tuberculosis, cutaneous" OR "tuberculosis, endocrine" "tuberculosis, gastrointestinal" OR "tuberculosis, hepatic" OR "tuberculosis, ocular" OR "tuberculosis, oral" OR "tuberculosis, osteoarticular" OR "tuberculosis, pleural" OR "tuberculosis, splenic" OR "tuberculosis, urogenital" OR "tuberculosis" AND "HIV" OR "AIDS" OR "human immunodeficiency virus" OR "acquired immunodeficiency syndrome" OR "co-infection" OR "opportunistic infection" OR "opportunistic" OR "sub Saharan Africa”.

\section{Methods of EPTB screening or diagnosis among PLWHA}

WHO or National TB Control Program diagnostic methods of the respective country were used; either combination or individual screening methods were used; either of the following methods were used: microbiologically determined sputum microscopy or sputum culture, PCR, GeneXpert, X-ray, broncho-alveolar lavage, histopathology, and ultrasound. Clinical signs and symptoms and response to treatment in combination with one of the abovementioned diagnostic methods were also used. In addition, methods of HIV screening or diagnosis as per WHO or national HIV screening or diagnosis protocol which mainly either rapid test or ELISA test or both were used.

\section{Methodological quality assessment}

All articles selected for inclusion in the review were subjected to a rigorous, independent appraisal by the investigators prior to inclusion in the review using standardized critical appraisal instruments from the Joanna Briggs Institute (JBI). ${ }^{13}$ Two reviewers independently appraised the studies. Disagreement was resolved by consensus between the two reviewers or through consultation with the third author, if required. The checklists were focused on setting, method of sampling techniques and sample size determination, screening/diagnostic method, and validity and reliability of measurements and data analysis. All relevant studies with optimum score were included and discussed with its limitations and risk of biases and how that might affect the results of this review.

\section{Study selection}

After studies were identified, we selected studies by passing through four steps primarily screening through titles and abstracts with over inclusion; second, full-text review for eligibility checkup was performed. Third, methodological critical appraisal was performed, and finally studies having optimum quality were included for systemic review and meta-analysis.

\section{Data collection process: data item and extraction tools}

We used a standardized form of JBI for data item and extraction. ${ }^{13}$ This data extraction form was used to extract the relevant information from appraised articles. A number of variables including study locations or country, years of publications, study periods, study designs, number of patients included in the study, and prevalence of EPTB/HIV co-infection were extracted from all studies included in the review. For this review, we used summary estimate rather than individual patient-level data.

\section{Data analysis and statistical methods}

Prevalence of EPTB was calculated as a number of EPTB cases per total number of PLWHA. The prevalence data were identified and abstracted or calculated from the given data from respective studies. In studies, when the standard error (SE) was not reported, we calculated it from the prevalence by using the following formula: $\mathrm{SE}=\sqrt{\mathrm{P}}(1-\mathrm{P}) / \mathrm{N}$ and $95 \%$ $\mathrm{CI}=\mathrm{P} \pm 1.96 \times \mathrm{SE}$, where $\mathrm{P}$ is the prevalence, $\mathrm{N}$ is the sample size or included PLWHA.

Meta-analysis was done by using Review Manager Software (version 5.3; the Cochrane Collaboration, 2014) and Compressive Meta-analysis Software (version 2.2). Heterogeneity of studies was assessed using Cochran's $Q$ (reported as $\chi^{2}, P$-values, and degrees of freedom $[d f]$ ) and $I^{2}$ statistics, which described the percentage of total variation between studies that were due to inconsistency rather than chance. ${ }^{14}$ Unlike $Q, I^{2}$ is inherently dependent on the number of studies included in the review: its values of $25 \%, 50 \%$, and $75 \%$ show low, moderate, and high degrees of heterogeneity, respectively. We also used inverse variance, random-effects model and proportion for the effect size estimate. Unlike fixed-effects model, random-effects model enabled us to see within and between study variations using Kendall's $\tau^{2} .{ }^{15}$ Sensitivity analysis and subgroup analysis were also performed. Funnel plot was used to see the possibility of publication bias and statistical tests of Egger's regression analysis and Begg's rank correlation analysis. ${ }^{16}$ We used forest plot to summarize the effect size. Finally, we used PRISMA standard for reporting. ${ }^{17}$ 

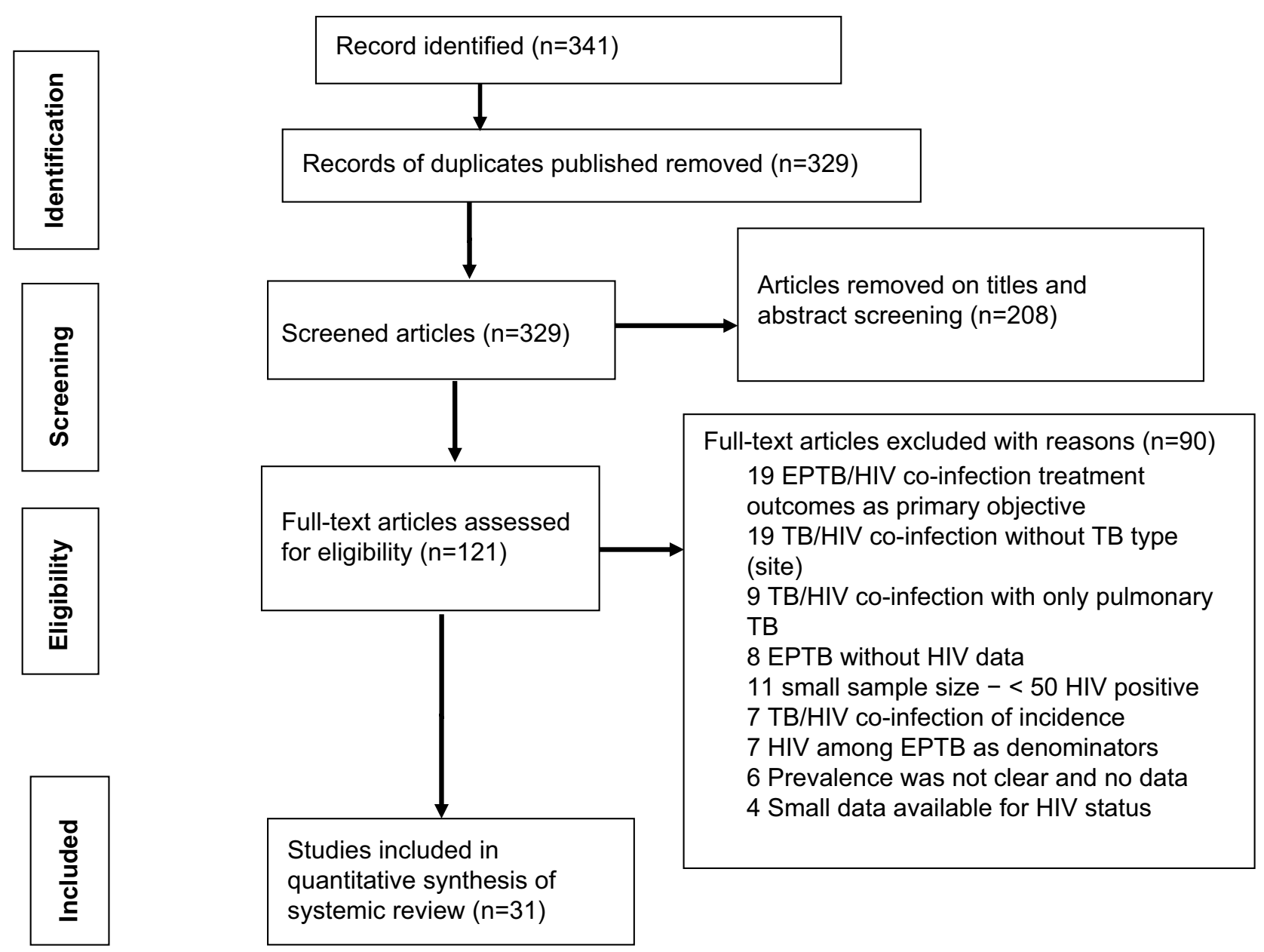

Figure I Study flow.

Notes: The number of studies and the sample size contributed by 12 countries from SSA that had included studies were as follows: ten studies with 2,837 people with HIV/AIDS were from Ethiopia, ${ }^{18-27}$ four each were from Nigeria $(2,042)^{28-31}$ and South Africa $(13,929),{ }^{32-35}$ and three were from Cameroon $(2,246)$. $^{11,36,37}$ Two each were from Uganda (245) ${ }^{38,39}$ and Zambia (47I), ${ }^{40,41}$ one each was from Tanzania (I75), ${ }^{42}$ Benin (52I), ${ }^{43}$ Burkina Faso (I I $),{ }^{44}$ Malawi (5,56I), ${ }^{45}$ Kenya (846), ${ }^{46}$ and Gabon (205) ${ }^{47}$ (Table I).

Abbreviations: EPTB, extrapulmonary TB; TB, tuberculosis.

\section{Results}

\section{Study characteristics}

In our search, we found 341 records. After the removal of duplicates and initial screening of titles and abstracts, we identified 121 potential full-text articles for eligibility. After screening the full-text articles, we included 31 studies in the review with 28,659 HIV-positive people (Figure 1).

\section{Methodological quality assessment of included studies}

The methodological quality assessments of studies were assessed by using JBI critical appraisal. As per the standard, the study that had optimum quality that was above the mean for "yes" was included (Table 2).

\section{Meta-analysis}

Our primary meta-analyses aimed to summarize the prevalence of EPTB among PLWHA.

On meta-analysis, data of 28,659 PLWHA as a sample were used to estimate the proportion of EPTB. The summary effect or pooled proportional estimate of meta-analysis of EPTB among PLWHA was found to be $20 \%$ (95\% CI: 17 , 22; heterogeneity: $\tau^{2}=0 ; \chi^{2}=509.09, d f=30, P<0.00001$; $I^{2}=94 \%$; Figure 2 ). 
Table I Characteristics of the studies included in the systemic review in SSA, 2017

\begin{tabular}{|c|c|c|c|c|c|c|c|c|}
\hline Study & Country & $\begin{array}{l}\text { Population } \\
\text { characteristics }\end{array}$ & Setting & EPTB investigation & Study types & Date & $\begin{array}{l}\text { Sample size } \\
\text { or total } \\
\text { included } \\
\text { (HIVIAIDS) }\end{array}$ & $\begin{array}{l}\text { Event } \\
\text { (EPTB) } \\
\text { (n) }\end{array}$ \\
\hline Ade et $\mathrm{al}^{43}$ & Benin & $\begin{array}{l}\text { All EPTB and new } \\
\text { PTB patients with or } \\
\text { without HIV and all } \\
\text { age groups }\end{array}$ & Hospital & $\begin{array}{l}\text { As per national } \\
\text { program }\end{array}$ & $\begin{array}{l}\text { Retrospective } \\
\text { cohort }\end{array}$ & $\begin{array}{l}\text { January to } \\
\text { December } \\
2011\end{array}$ & 521 & 72 \\
\hline Adejumo et $\mathrm{a}^{30}$ & Nigeria & $\begin{array}{l}\text { All TB patients with } \\
\text { or without HIV and } \\
\text { all age groups }\end{array}$ & UBTH & $\begin{array}{l}\text { Diagnosed as per } \\
\text { Nigerian national } \\
\text { program }\end{array}$ & $\begin{array}{l}\text { Retrospective } \\
\text { review of } \\
\text { treatment cards } \\
\text { of patients }\end{array}$ & $\begin{array}{l}\text { January I, } \\
2014, \text { to } \\
\text { December } \\
31,2014\end{array}$ & 72 & 10 \\
\hline Affusim et $\mathrm{a}^{29}$ & Nigeria & $\begin{array}{l}\text { All HIV-positive TB } \\
\text { patients, }>18 \text { years }\end{array}$ & $\begin{array}{l}\text { Teaching } \\
\text { hospital }\end{array}$ & $\begin{array}{l}\text { As per national } \\
\text { program }\end{array}$ & Cross-sectional & $\begin{array}{l}\text { January to } \\
\text { April } 2007\end{array}$ & 330 & 21 \\
\hline Agbor et al" & Cameroon & $\begin{array}{l}\text { All HIV/TB patients } \\
\text { and inpatients, aged } \\
\geq I 5 \text { years }\end{array}$ & Hospital & $\begin{array}{l}\text { As per national } \\
\text { program }\end{array}$ & Cross-sectional & $\begin{array}{l}\text { January I, } \\
2006, \text { to June } \\
30,2013\end{array}$ & 335 & 119 \\
\hline $\begin{array}{l}\text { Alemie and } \\
\text { Gebreselassie }^{18}\end{array}$ & Ethiopia & $\begin{array}{l}\text { All TB patients with } \\
\text { or without HIV and } \\
\text { all age groups }\end{array}$ & $\begin{array}{l}\text { Private } \\
\text { health } \\
\text { institution }\end{array}$ & $\begin{array}{l}\text { As per national } \\
\text { program }\end{array}$ & Cross-sectional & $\begin{array}{l}\text { In the year } \\
2008 / 2009\end{array}$ & 159 & 52 \\
\hline Amare ${ }^{19}$ & Ethiopia & $\begin{array}{l}\text { All children with TB } \\
\text { aged }<15 \text { years with } \\
\text { or without HIV }\end{array}$ & $\begin{array}{l}\text { Private, } \\
\text { public } \\
\text { (primary, } \\
\text { secondary, } \\
\text { tertiary) }\end{array}$ & $\begin{array}{l}\text { As per national } \\
\text { TB program, } \\
\text { for histological } \\
\text { and radiological } \\
\text { performance }\end{array}$ & $\begin{array}{l}\text { Retrospective } \\
\text { cohort }\end{array}$ & $\begin{array}{l}\text { January I, } \\
2012 \text {, to } \\
\text { December } \\
31,2013\end{array}$ & 133 & 49 \\
\hline $\begin{array}{l}\text { Gebreegziabher } \\
\text { et } \mathrm{al}^{20}\end{array}$ & Ethiopia & $\begin{array}{l}\text { All TB patients with } \\
\text { or without HIV and } \\
\text { all age groups }\end{array}$ & $\begin{array}{l}30 \text { public } \\
\text { health } \\
\text { facilities }\end{array}$ & $\begin{array}{l}\text { As per national } \\
\text { guidelines }\end{array}$ & $\begin{array}{l}\text { Retrospective } \\
\text { cohort study }\end{array}$ & $\begin{array}{l}\text { July } 2007 \text { and } \\
\text { June } 2012\end{array}$ & 753 & 249 \\
\hline Daniel et $\mathrm{a}^{28}$ & Nigeria & $\begin{array}{l}\text { All TB patients with } \\
\text { or without HIV and } \\
\text { all age groups }\end{array}$ & Hospital & $\begin{array}{l}\text { Culture and/or } \\
\text { sputum smear, rapid } \\
\text { test for HIV }\end{array}$ & $\begin{array}{l}\text { Retrospective } \\
\text { review }\end{array}$ & $\begin{array}{l}\text { January I, } \\
\text { I99I, to } \\
\text { December 31, } \\
\text { 199| }\end{array}$ & 320 & 23 \\
\hline Deribew et $\mathrm{a}^{21}$ & Ethiopia & $\begin{array}{l}\text { All adult } \geq 15 \text {-year } \\
\text { TB patients with or } \\
\text { without HIV }\end{array}$ & $\begin{array}{l}\text { Health } \\
\text { centers }\end{array}$ & $\begin{array}{l}\text { TB lymphadenitis was } \\
\text { diagnosed based on } \\
\text { clinical parameters } \\
\text { and cytological } \\
\text { examination }\end{array}$ & Cross-sectional & $\begin{array}{l}\text { February } \\
2009 \text { to } \\
\text { March } 2009\end{array}$ & 81 & 10 \\
\hline Esmael et $\mathrm{a}^{22}$ & Ethiopia & $\begin{array}{l}\text { All TB patients with } \\
\text { or without HIV and } \\
\text { all age groups }\end{array}$ & $\begin{array}{l}\text { Referral } \\
\text { hospital }\end{array}$ & $\begin{array}{l}\text { AFB, chest } \\
\text { radiographs, and for } \\
\text { EPTB, pathological } \\
\text { examination }\end{array}$ & $\begin{array}{l}\text { Retrospective } \\
\text { record review }\end{array}$ & $\begin{array}{l}\text { June } 2008 \text { to } \\
\text { August } 2013\end{array}$ & 321 & 51 \\
\hline Feldacker et $\mathrm{al}^{45}$ & Malawi & $\begin{array}{l}\text { All TB patients with } \\
\text { or without HIV and } \\
\text { all age groups }\end{array}$ & $\begin{array}{l}\text { TB/HIV } \\
\text { integrated } \\
\text { clinic }\end{array}$ & $\begin{array}{l}\text { Bacteriological or } \\
\text { histopathological, opt- } \\
\text { out HIV testing }\end{array}$ & $\begin{array}{l}\text { Retrospective } \\
\text { cohort }\end{array}$ & $\begin{array}{l}\text { January } 2008 \\
\text { to December } \\
2010\end{array}$ & 5,561 & 1,117 \\
\hline Gupta et $\mathrm{al}^{32}$ & $\begin{array}{l}\text { South } \\
\text { Africa }\end{array}$ & $\begin{array}{l}\text { All TB patients with } \\
\text { or without HIV and } \\
\text { all age groups }\end{array}$ & $\begin{array}{l}\text { Electronic } \\
\text { TB registry }\end{array}$ & $\begin{array}{l}\text { ICD-I0 classification } \\
\text { used for EPTB, } \\
\text { serology for HIV }\end{array}$ & $\begin{array}{l}\text { Retrospective } \\
\text { analysis }\end{array}$ & 2009 & 13,237 & 2,328 \\
\hline Hailu et $\mathrm{a}^{23}$ & Ethiopia & $\begin{array}{l}\text { All children with TB } \\
\text { aged }<15 \text { years with } \\
\text { or without HIV }\end{array}$ & $\begin{array}{l}\text { Health } \\
\text { centers }\end{array}$ & $\begin{array}{l}\text { Clinical history, chest } \\
\text { X-ray, HIV testing, } \\
\text { and histopathology }\end{array}$ & $\begin{array}{l}\text { Retrospective } \\
\text { study }\end{array}$ & $\begin{array}{l}\text { January } 2007 \\
\text { to December } \\
2011\end{array}$ & 346 & 103 \\
\hline Hoffmann et $\mathrm{a}^{33}$ & $\begin{array}{l}\text { South } \\
\text { Africa }\end{array}$ & $\begin{array}{l}\text { All adult }>18 \text {-year } \\
\text { HIV-positive patients } \\
\text { with liver TB }\end{array}$ & Hospital & $\begin{array}{l}\text { Clinical, laboratory, } \\
\text { and radiography }\end{array}$ & $\begin{array}{l}\text { Retrospective } \\
\text { study }\end{array}$ & $200 I-2009$ & 108 & 28 \\
\hline $\begin{array}{l}\text { Iliyasu and } \\
\text { Babashani }{ }^{31}\end{array}$ & Nigeria & $\begin{array}{l}\text { All HIV-positive } \\
\text { patients with or } \\
\text { without TB and all } \\
\text { age groups }\end{array}$ & $\begin{array}{l}\text { Teaching } \\
\text { hospital }\end{array}$ & $\begin{array}{l}\text { Biopsy of tissue } \\
\text { specimen, TB culture }\end{array}$ & $\begin{array}{l}\text { Retrospective } \\
\text { review of } \\
\text { charts }\end{array}$ & $\begin{array}{l}\text { January } 2006 \\
\text { to December } \\
2006\end{array}$ & 138 & 50 \\
\hline
\end{tabular}

(Continued) 
Table I (Continued)

\begin{tabular}{|c|c|c|c|c|c|c|c|c|}
\hline Study & Country & $\begin{array}{l}\text { Population } \\
\text { characteristics }\end{array}$ & Setting & EPTB investigation & Study types & Date & $\begin{array}{l}\text { Sample size } \\
\text { or total } \\
\text { included } \\
\text { (HIVIAIDS) }\end{array}$ & $\begin{array}{l}\text { Event } \\
\text { (EPTB) } \\
\text { (n) }\end{array}$ \\
\hline $\begin{array}{l}\text { Kamenju and } \\
\text { Said }^{42}\end{array}$ & Tanzania & $\begin{array}{l}\text { All TB patients with } \\
\text { or without HIV and } \\
\text { all age groups }\end{array}$ & Hospital & $\begin{array}{l}\text { Smear, radiological } \\
\text { tests, culture, biopsy, } \\
\text { or a combination }\end{array}$ & $\begin{array}{l}\text { Retrospective } \\
\text { cross-sectional } \\
\text { analyses }\end{array}$ & $\begin{array}{l}\text { August } 2008 \\
\text { to July } 2009\end{array}$ & 175 & 19 \\
\hline Malkin et $\mathrm{al}^{44}$ & $\begin{array}{l}\text { Burkina } \\
\text { Faso }\end{array}$ & $\begin{array}{l}\text { All TB patients with } \\
\text { or without HIV and } \\
\text { all age groups }\end{array}$ & TB center & $\begin{array}{l}\text { Smear, radiological } \\
\text { tests, culture, biopsy, } \\
\text { or a combination }\end{array}$ & Cohort study & $\begin{array}{l}\text { October } 1988 \\
\text { to March } \\
1992\end{array}$ & 112 & 22 \\
\hline $\begin{array}{l}\text { Mekonnen } \\
\text { et } \mathrm{al}^{24}\end{array}$ & Ethiopia & $\begin{array}{l}\text { All TB patients with } \\
\text { or without HIV and } \\
\text { all age groups }\end{array}$ & $\begin{array}{l}\text { Hospital } \\
\text { and private } \\
\text { clinic }\end{array}$ & $\begin{array}{l}\text { Clinical history with } \\
\text { X-ray, ultrasound, } \\
\text { and/or pathological } \\
\text { techniques }\end{array}$ & $\begin{array}{l}\text { Retrospective } \\
\text { study }\end{array}$ & $\begin{array}{l}\text { January 20II } \\
\text { to December } \\
2014\end{array}$ & 236 & 78 \\
\hline Muttai et $\mathrm{al}^{46}$ & Kenya & $\begin{array}{l}\text { Adult } \geq 15 \text {-year TB } \\
\text { patients diagnosed } \\
\text { with } \mathrm{HIV}+\end{array}$ & $\begin{array}{l}\text { Hospitals } \\
\text { and health } \\
\text { centers }\end{array}$ & Not reported & $\begin{array}{l}\text { Retrospective } \\
\text { chart review }\end{array}$ & $\begin{array}{c}\text { October } 2006 \\
\text { to April } 2008\end{array}$ & 846 & 145 \\
\hline Namme et $\mathrm{al}^{36}$ & Cameroon & $\begin{array}{l}\text { All TB patients with } \\
\text { or without HIV and } \\
\text { all age groups }\end{array}$ & Hospital & $\begin{array}{l}\text { Smear, radiological } \\
\text { tests, biopsy, or } \\
\text { combination }\end{array}$ & Cross-sectional & $\begin{array}{l}\text { July I, } 2007 \text {, } \\
\text { to June } 30, \\
201 \mathrm{I}\end{array}$ & 438 & 108 \\
\hline Njozing et $\mathrm{al}^{37}$ & Cameroon & $\begin{array}{l}\text { All TB patients with } \\
\text { or without HIV and } \\
\text { all age groups }\end{array}$ & $\begin{array}{l}\text { TB/HIV } \\
\text { treatment } \\
\text { center }\end{array}$ & $\begin{array}{l}\text { Clinical and/or } \\
\text { radiological, biopsies } \\
\text { and laboratory } \\
\text { examination of } \\
\text { aspirates from } \\
\text { affected areas }\end{array}$ & $\begin{array}{l}\text { Retrospective } \\
\text { cohort }\end{array}$ & $\begin{array}{l}\text { January } 2006 \\
\text { to December } \\
2007\end{array}$ & 1,473 & 206 \\
\hline Ocama et $\mathrm{al}^{38}$ & Uganda & $\begin{array}{l}\text { All HIV-positive } \\
\text { patients who } \\
\text { suspected for liver } \\
\text { disease }\end{array}$ & $\begin{array}{l}\text { Teaching } \\
\text { hospital }\end{array}$ & $\begin{array}{l}\text { Stool analysis, } \\
\text { ultrasound-guided } \\
\text { liver biopsies }\end{array}$ & Cross-sectional & $\begin{array}{l}\text { May } 2004 \text { to } \\
\text { March } 2005\end{array}$ & 77 & 7 \\
\hline Nglazi et $\mathrm{al}^{34}$ & $\begin{array}{l}\text { South } \\
\text { Africa }\end{array}$ & $\begin{array}{l}\text { All TB patients with } \\
\text { or without HIV aged } \\
\geq 15 \text { years }\end{array}$ & Hospital & $\begin{array}{l}\text { As per national } \\
\text { guidelines }\end{array}$ & $\begin{array}{l}\text { Retrospective } \\
\text { cohort study }\end{array}$ & $\begin{array}{l}\text { October } 2009 \\
\text { and June 201 I }\end{array}$ & 472 & 159 \\
\hline Siddiqi et al ${ }^{40}$ & Zambia & $\begin{array}{l}\text { All HIV-positive } \\
\text { patients with a CNS } \\
\text { infection requiring } \\
\text { a LP }\end{array}$ & $\begin{array}{l}\text { Teaching } \\
\text { hospital }\end{array}$ & $\begin{array}{l}\text { Receiving an LP } \\
\text { microscopy, culture, } \\
\text { Gram stain, and India } \\
\text { ink testing on CSF }\end{array}$ & $\begin{array}{l}\text { Cross-sectional } \\
\text { study }\end{array}$ & $\begin{array}{l}\text { October I, } \\
2010 \text { to May } \\
31,2012\end{array}$ & 331 & 48 \\
\hline Skogmar et $\mathrm{al}^{25}$ & Ethiopia & $\begin{array}{l}\text { All TB patients with } \\
\text { or without HIV and } \\
\geq 18 \text { years old }\end{array}$ & $\begin{array}{l}\text { Hospitals } \\
\text { and health } \\
\text { centers }\end{array}$ & $\begin{array}{l}\text { As per national } \\
\text { program }\end{array}$ & $\begin{array}{l}\text { Cross-sectional } \\
\text { study }\end{array}$ & $\begin{array}{l}\text { September } \\
2010 \text { to } \\
\text { March } 2012\end{array}$ & 307 & 91 \\
\hline $\begin{array}{l}\text { Tarekegne } \\
\text { et } \mathrm{al}^{26}\end{array}$ & Ethiopia & $\begin{array}{l}\text { All TB patients with } \\
\text { or without HIV and } \\
\text { all age groups }\end{array}$ & Hospital & $\begin{array}{l}\text { Pathological } \\
\text { examination and/or } \\
\text { clinical symptoms }\end{array}$ & $\begin{array}{l}\text { Retrospective } \\
\text { study }\end{array}$ & $\begin{array}{l}\text { September } \\
2009 \text { to } \\
\text { August } 2012\end{array}$ & 404 & 58 \\
\hline $\begin{array}{l}\text { Wobudeya } \\
\text { et } \text { al }^{39}\end{array}$ & Uganda & $\begin{array}{l}\text { All children with TB } \\
\text { aged }<15 \text { years with } \\
\text { or without HIV }\end{array}$ & Five districts & $\begin{array}{l}\text { As per national } \\
\text { program and } \mathrm{WHO}\end{array}$ & $\begin{array}{l}\text { Retrospective } \\
\text { cross-sectional } \\
\text { study }\end{array}$ & $\begin{array}{l}\text { January I, } \\
2009, \text { to } \\
\text { December } 31, \\
2010\end{array}$ & 168 & 24 \\
\hline Yassin et $\mathrm{al}^{27}$ & Ethiopia & $\begin{array}{l}\text { All TB patients with } \\
\text { or without HIV and } \\
\text { all age groups }\end{array}$ & $\begin{array}{l}\text { District } \\
\text { hospital }\end{array}$ & $\begin{array}{l}\text { Sputum microscopy, } \\
\text { X-ray facilities }\end{array}$ & $\begin{array}{l}\text { Prospective } \\
\text { cohort }\end{array}$ & Not reported & 97 & 10 \\
\hline Sinkala et $\mathrm{al}^{41}$ & Zambia & $\begin{array}{l}\text { All abdominal TB } \\
\text { and HIV positive }\end{array}$ & $\begin{array}{l}\text { University } \\
\text { Teaching } \\
\text { Hospital, } \\
\text { Lusaka, } \\
\text { Zambia }\end{array}$ & $\begin{array}{l}\text { Culture andlor } \\
\text { granulomatous on } \\
\text { histopathological } \\
\text { examination } \\
\text { microscopy }\end{array}$ & $\begin{array}{l}\text { Cross-sectional } \\
\text { study }\end{array}$ & $\begin{array}{l}\text { September } \\
2005 \text { until } \\
\text { May } 2006\end{array}$ & 140 & 22 \\
\hline Stolp et $\mathrm{al}^{47}$ & Gabon & $\begin{array}{l}\text { All TB with or } \\
\text { without HIV and all } \\
\text { age groups }\end{array}$ & $\begin{array}{l}\text { Albert } \\
\text { Schweitzer } \\
\text { Hospital, } \\
\text { Lambarene, } \\
\text { Gabon }\end{array}$ & $\begin{array}{l}\text { Neelsen-stained } \\
\text { sputum smear or on } \\
\text { clinical grounds, HIV } \\
\text { test }\end{array}$ & $\begin{array}{l}\text { Retrospective } \\
\text { study }\end{array}$ & $\begin{array}{l}\text { January } 2007 \\
\text { to December } \\
2012\end{array}$ & 205 & 40 \\
\hline
\end{tabular}

(Continued) 
Table I (Continued)

\begin{tabular}{|c|c|c|c|c|c|c|c|c|}
\hline Study & Country & $\begin{array}{l}\text { Population } \\
\text { characteristics }\end{array}$ & Setting & EPTB investigation & Study types & Date & $\begin{array}{l}\text { Sample size } \\
\text { or total } \\
\text { included } \\
\text { (HIVIAIDS) }\end{array}$ & $\begin{array}{l}\text { Event } \\
\text { (EPTB) } \\
\text { (n) }\end{array}$ \\
\hline $\begin{array}{l}\text { van der Plas } \\
\text { et } \mathrm{al}^{35}\end{array}$ & $\begin{array}{l}\text { South } \\
\text { Africa }\end{array}$ & $\begin{array}{l}\text { Adults ( }>18 \text { years), } \\
\text { hospitalized HIV } \\
\text { and PTB or EPTB } \\
\text { patients, not on ART }\end{array}$ & $\begin{array}{l}\mathrm{BCH} \text {, Cape } \\
\text { town }\end{array}$ & $\begin{array}{l}\text { Smear or culture, } \\
\text { WHO guidelines, } \\
\text { smear negative or } \\
\text { EPTB in HIV-I- } \\
\text { infected people }\end{array}$ & $\begin{array}{l}\text { Prospective } \\
\text { observational } \\
\text { cohort }\end{array}$ & $\begin{array}{l}\text { April 29, } \\
\text { 2009, until } \\
\text { February 28, } \\
2011\end{array}$ & 112 & 20 \\
\hline
\end{tabular}

Abbreviations: AFB, acid-fast bacilli; ART, antiretroviral therapy; BCH, Brooklyn Chest Hospital; CNS, central nervous system; CSF, cerebrospinal fluid; EPTB, extrapulmonary TB; LP, lumbar puncture; PTB, pulmonary TB; SSA, sub-Saharan Africa; TB, tuberculosis; UBTH, University of Benin Teaching Hospital.

Table 2 Critical appraisal results of studies of EPTB among PLWHA by using JBI prevalence critical appraisal checklist, SSA, 2017

\begin{tabular}{|c|c|c|c|c|c|c|c|c|c|c|c|c|c|}
\hline \multirow[t]{2}{*}{ Study } & \multirow[t]{2}{*}{ QI } & \multirow[t]{2}{*}{ Q2 } & \multirow[t]{2}{*}{ Q3 } & \multirow[t]{2}{*}{ Q4 } & \multirow[t]{2}{*}{ Q5 } & \multirow[t]{2}{*}{ Q6 } & \multirow[t]{2}{*}{ Q7 } & \multirow[t]{2}{*}{ Q8 } & \multirow[t]{2}{*}{ Q9 } & \multirow[t]{2}{*}{ Q10 } & \multicolumn{3}{|c|}{ Total } \\
\hline & & & & & & & & & & & $\mathbf{Y}$ & $\mathbf{N}$ & $\mathbf{u}$ \\
\hline Ade et $\mathrm{al}^{43}$ & $Y$ & $Y$ & $Y$ & $Y$ & $Y$ & $Y$ & $Y$ & $\mathrm{Y}$ & $\mathrm{N}$ & $Y$ & 9 & 1 & 0 \\
\hline Adejumo et $\mathrm{al}^{30}$ & $\mathrm{~N}$ & $\mathrm{~N}$ & $U$ & Y & Y & $Y$ & $Y$ & $Y$ & $\mathrm{~N}$ & $N$ & 5 & 4 & I \\
\hline Affusim et $\mathrm{al}^{29}$ & $Y$ & Y & Y & Y & Y & Y & $Y$ & $Y$ & $Y$ & $U$ & 9 & 0 & I \\
\hline Agbor et al" & $Y$ & Y & Y & Y & Y & Y & Y & Y & Y & Y & 10 & 0 & 0 \\
\hline Alemie and Gebreselassie ${ }^{18}$ & $U$ & Y & $\mathrm{N}$ & $U$ & Y & Y & $\mathrm{U}$ & $Y$ & $Y$ & $Y$ & 6 & 2 & 2 \\
\hline Amare ${ }^{19}$ & $Y$ & Y & Y & $Y$ & Y & $\mathrm{N}$ & $\mathrm{N}$ & $\mathrm{Y}$ & $Y$ & $Y$ & 8 & 2 & 0 \\
\hline Gebreegziabher et $\mathrm{a}^{20}$ & $Y$ & Y & Y & Y & $U$ & $Y$ & $Y$ & $Y$ & $\mathrm{Y}$ & $Y$ & 9 & 0 & 1 \\
\hline Daniel et $\mathrm{a}^{28}$ & $Y$ & $Y$ & $Y$ & $Y$ & $Y$ & $Y$ & $Y$ & $Y$ & $Y$ & $Y$ & 10 & 0 & 0 \\
\hline Deribew et $\mathrm{al}^{21}$ & $Y$ & $\mathrm{~N}$ & Y & $Y$ & $Y$ & $Y$ & $Y$ & $Y$ & $\mathrm{Y}$ & $Y$ & 9 & 1 & 0 \\
\hline Esmael et $\mathrm{al}^{22}$ & $Y$ & Y & Y & Y & Y & $Y$ & Y & $Y$ & $Y$ & Y & 10 & 0 & 0 \\
\hline Feldacker et a $\left.\right|^{45}$ & $Y$ & Y & Y & $Y$ & Y & $Y$ & $Y$ & $Y$ & $\mathrm{Y}$ & $Y$ & 10 & 0 & 0 \\
\hline Gupta et $\mathrm{al}^{32}$ & $Y$ & Y & Y & $Y$ & Y & $Y$ & $Y$ & $Y$ & $\mathrm{Y}$ & $Y$ & 10 & 0 & 0 \\
\hline Hailu et $\mathrm{a}^{23}$ & $Y$ & $U$ & Y & Y & $Y$ & $Y$ & $Y$ & $Y$ & Y & $Y$ & 9 & 0 & 1 \\
\hline Hoffmann et $\mathrm{a}^{33}$ & $Y$ & Y & Y & $Y$ & $Y$ & $Y$ & $Y$ & $Y$ & Y & Y & 10 & 0 & 0 \\
\hline Iliyasu and Babashani' & Y & Y & Y & Y & Y & $Y$ & $Y$ & $Y$ & $N$ & $Y$ & 9 & 1 & 0 \\
\hline Kamenju and Said ${ }^{42}$ & $Y$ & Y & $\mathrm{N}$ & $Y$ & $Y$ & $Y$ & $Y$ & $Y$ & $U$ & $Y$ & 8 & 1 & I \\
\hline Malkin et $\mathrm{a}^{44}$ & $Y$ & Y & Y & $Y$ & $Y$ & $Y$ & $Y$ & $Y$ & $Y$ & $Y$ & 10 & 0 & 0 \\
\hline Mekonnen et $\mathrm{a}^{24}$ & $Y$ & $U$ & $U$ & Y & $Y$ & $Y$ & $Y$ & $Y$ & U & $Y$ & 7 & 0 & 3 \\
\hline Muttai et $\mathrm{a}^{46}$ & $Y$ & Y & $U$ & Y & $Y$ & $Y$ & $Y$ & $Y$ & $Y$ & $Y$ & 9 & 0 & I \\
\hline Namme et $a^{36}$ & $Y$ & $\mathrm{~N}$ & $U$ & $Y$ & $Y$ & $Y$ & $Y$ & $Y$ & $Y$ & $Y$ & 8 & 1 & I \\
\hline Njozing et $\mathrm{al}^{37}$ & $Y$ & Y & Y & $Y$ & $Y$ & $\mathrm{~N}$ & $N$ & $Y$ & $Y$ & $Y$ & 8 & 2 & 0 \\
\hline Ocama et $\mathrm{a}^{38}$ & $Y$ & Y & Y & $Y$ & $Y$ & $Y$ & $Y$ & $Y$ & $Y$ & $Y$ & 10 & 0 & 0 \\
\hline Nglazi et a $\left.\right|^{34}$ & $Y$ & Y & Y & $Y$ & $Y$ & $Y$ & $Y$ & $Y$ & Y & $Y$ & 10 & 0 & 0 \\
\hline Siddiqi et $\mathrm{a}^{40}$ & $Y$ & Y & $U$ & $Y$ & $Y$ & $Y$ & $Y$ & $Y$ & $Y$ & Y & 9 & 0 & 1 \\
\hline Skogmar et $\mathrm{al}^{25}$ & $Y$ & Y & $Y$ & $Y$ & $Y$ & $Y$ & $Y$ & $Y$ & $Y$ & $Y$ & 10 & 0 & 0 \\
\hline Tarekegne et $\mathrm{al}^{26}$ & $Y$ & Y & $Y$ & $Y$ & $Y$ & $Y$ & $Y$ & $Y$ & $Y$ & $Y$ & 10 & 0 & 0 \\
\hline Wobudeya et $\mathrm{a}^{39}$ & $Y$ & $Y$ & Y & $Y$ & $Y$ & $Y$ & $Y$ & $Y$ & $Y$ & $Y$ & 10 & 0 & 0 \\
\hline Yassin et $\mathrm{a}^{27}$ & $Y$ & Y & $U$ & Y & Y & $Y$ & $Y$ & $Y$ & $Y$ & $Y$ & 9 & 0 & I \\
\hline Sinkala et $\mathrm{al}^{41}$ & Y & $Y$ & Y & Y & $Y$ & $Y$ & $Y$ & $Y$ & $Y$ & $Y$ & 10 & 0 & 0 \\
\hline Stolp et $\mathrm{a}^{47}$ & $Y$ & Y & Y & Y & $Y$ & $Y$ & $Y$ & $Y$ & U & $Y$ & 9 & 0 & 1 \\
\hline van der Plas et $a^{35}$ & $Y$ & Y & $U$ & $Y$ & $Y$ & $Y$ & $Y$ & $Y$ & $Y$ & $Y$ & 9 & 0 & 0 \\
\hline
\end{tabular}

Abbreviations: EPTB, extrapulmonary tuberculosis; JBI, Joanna Briggs Institute; N, number; PLWHA, people living with HIV/AIDS; Q, question; SSA, sub-Saharan Africa; $\mathrm{U}$, unclear; $\mathrm{Y}$, yes.

\section{Sensitivity analysis}

For the statistical fidelity, sensitivity analysis was performed using Compressive Meta-Analysis Software by removing one study while keeping the others in random-effects model, and by excluding one large study, ${ }^{32}$ the prevalence estimate of EPTB among PLWHA was not changed, $20 \%$ (95\% CI: 17 ,
23; heterogeneity: $\tau^{2}=0.01 ; \chi^{2}=507.37, d f=29, P<0.00001$; $r^{2}=94 \%$; Table 3 ).

\section{Subgroup analysis}

Subgroup analysis was done to find heterogeneity based on age, types of EPTB, study design, subregion of SSA, and 


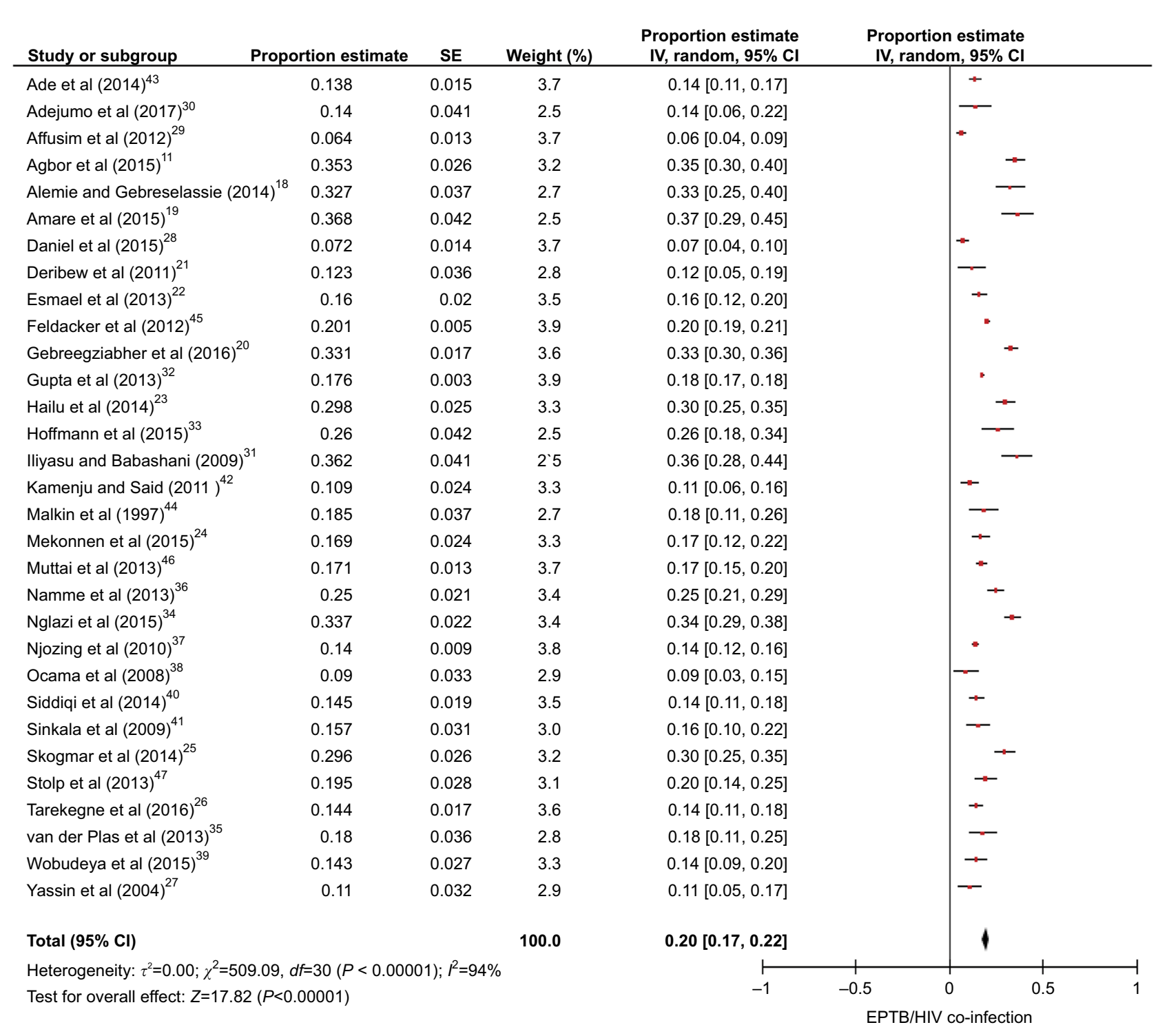

Figure 2 Forest plot including all available relevant studies on EPTB among PLWHA in SSA, 2017.

Abbreviations: $d f$, degrees of freedom; EPTB, extrapulmonary tuberculosis; PLWHA, people living with HIVIAIDS; SE, standard error; SSA, sub-Saharan Africa.

countries having two or more studies and the time variation. For instance, by study population age group, inconsistency of the estimate was not removed, the EPTB among PLWHA did not vary by age group when we compared children $<15$ years, ${ }^{23,28,39}$ adults $\geq 15$ years ${ }^{11,21,46}$ or $\geq 18$ years ${ }^{25,29,33}$ and all age groups in remaining 21 studies (Table 3 ).

\section{Effect of publication year on estimate}

To determine the effect of year of publications on EPTB prevalence estimate among PLWHA, we performed metaregression using Compressive Meta-analysis (CMA) Software. We observed that publication year increased by a year and the point estimate of EPTB prevalence among PLWHA increased with $0.4 \%$ at $95 \% \mathrm{CI}(0.2,0.6 \%), P=0.00067$ (Figure 3).

\section{Publication bias}

We assessed publication bias using CMA Software through funnel plot and regression tests with random-effects model for included studies (Figure 4). There was no publication bias. In addition, we performed statistical test, and no evidence of publication bias was observed ( $P=0.44$ for Egger's regression analysis; $P=0.11$ for Begg's rank correlation analysis). 
Table 3 Heterogeneity assessment summary by using sensitivity and subgroup analyses, SSA, 2017

\begin{tabular}{|c|c|c|c|c|c|}
\hline \multirow[t]{2}{*}{ Subgroup analysis (PICO) } & \multirow{2}{*}{$\begin{array}{l}\text { Number } \\
\text { of studies }\end{array}$} & \multirow{2}{*}{$\begin{array}{l}\text { Prevalence of EPTB }(95 \% \mathrm{Cl}) \text {, } \\
\text { random-effects model }\end{array}$} & \multicolumn{3}{|c|}{ Heterogeneity } \\
\hline & & & $\tau^{2}$ & $P^{2}(\%)$ & $P$-value (Q) \\
\hline Overall & 31 & $20 \%(17,22)$ & 0 & 94 & $<0.00001$ \\
\hline Sensitivity analysis by removing one large study & 30 & $20 \%(17,23)$ & 0.01 & 94 & $<0.00001$ \\
\hline \multicolumn{6}{|l|}{ Clinical heterogeneity by age groups } \\
\hline Children $<15$ years & 3 & $17 \%(3,31)$ & 0.01 & 97 & $<0.0000$ I \\
\hline Adults $>15$ or $>18$ years & 7 & $23 \%(14,32)$ & 0.01 & 97 & $<0.0000$ I \\
\hline All age groups & 21 & $18 \%(16,19)$ & 0 & 86 & $<0.00001$ \\
\hline \multicolumn{6}{|l|}{ Clinical heterogeneity by EPTB types } \\
\hline Different EPTB & 27 & $22 \%(19,25)$ & 0.01 & 97 & $<0.00001$ \\
\hline TB of lymph node & 2 & $14 \%(12,16)$ & 0 & 0 & 0.65 \\
\hline TB of hepatic or TB of abdomen & 2 & One each, not estimated & & & \\
\hline \multicolumn{6}{|l|}{ Methodological heterogeneity by study design } \\
\hline Cross-sectional & 9 & $20 \%(13,27)$ & 0.01 & 96 & $<0.00001$ \\
\hline Retrospective record review & 14 & $19 \%(16,22)$ & 0 & 91 & $<0.0000$ I \\
\hline Retrospective cohort & 5 & $23 \%(17,29)$ & 0 & 97 & $<0.00001$ \\
\hline Prospective cohort & 2 & $16 \%(11,20)$ & 0 & 36 & 0.21 \\
\hline \multicolumn{6}{|l|}{ By subregion of SSA } \\
\hline Eastern SSA & 16 & $20 \%(16,23)$ & 0 & 92 & $<0.0000$ I \\
\hline Southern SSA & 4 & $21 \%(15,26)$ & 0 & 92 & $<0.0000$ I \\
\hline Western SSA & 10 & $19 \%(13,24)$ & 0.01 & 96 & $<0.0000$ I \\
\hline Central SSA & 1 & Not estimated & & & \\
\hline \multicolumn{6}{|l|}{ By countries of SSA having $\geq$ two studies } \\
\hline Ethiopia & 10 & $23 \%(17,29)$ & 0.01 & 93 & $<0.0000$ I \\
\hline Nigeria & 4 & $15 \%(7,24)$ & 0.01 & 94 & $<0.00001$ \\
\hline Cameroon & 3 & $25 \%(12,37)$ & 0.01 & 97 & $<0.0000$ I \\
\hline South Africa & 4 & $17 \%(14,20)$ & 0 & 84 & 0.0003 \\
\hline Uganda & 2 & $12 \%(7,17)$ & 0 & 35 & 0.21 \\
\hline Zambia & 2 & $15 \%(12,18)$ & 0 & 0 & 0.74 \\
\hline Others & 6 & $17 \%(14,20)$ & 0 & 84 & $<0.0001$ \\
\hline \multicolumn{6}{|l|}{ Study time variation } \\
\hline $1990-2000$ & 1 & Not estimated & & & \\
\hline $200 I-2010$ & 3 & $19 \%(3,34)$ & 0.02 & 94 & $<0.00001$ \\
\hline $2011-2017$ & 27 & $20 \%(18,23)$ & 0 & 94 & $<0.00001$ \\
\hline
\end{tabular}

Abbreviations: EPTB, extrapulmonary tuberculosis; PICO, people, intervention, comparison, outcomes; SSA, sub-Saharan Africa; TB, tuberculosis.

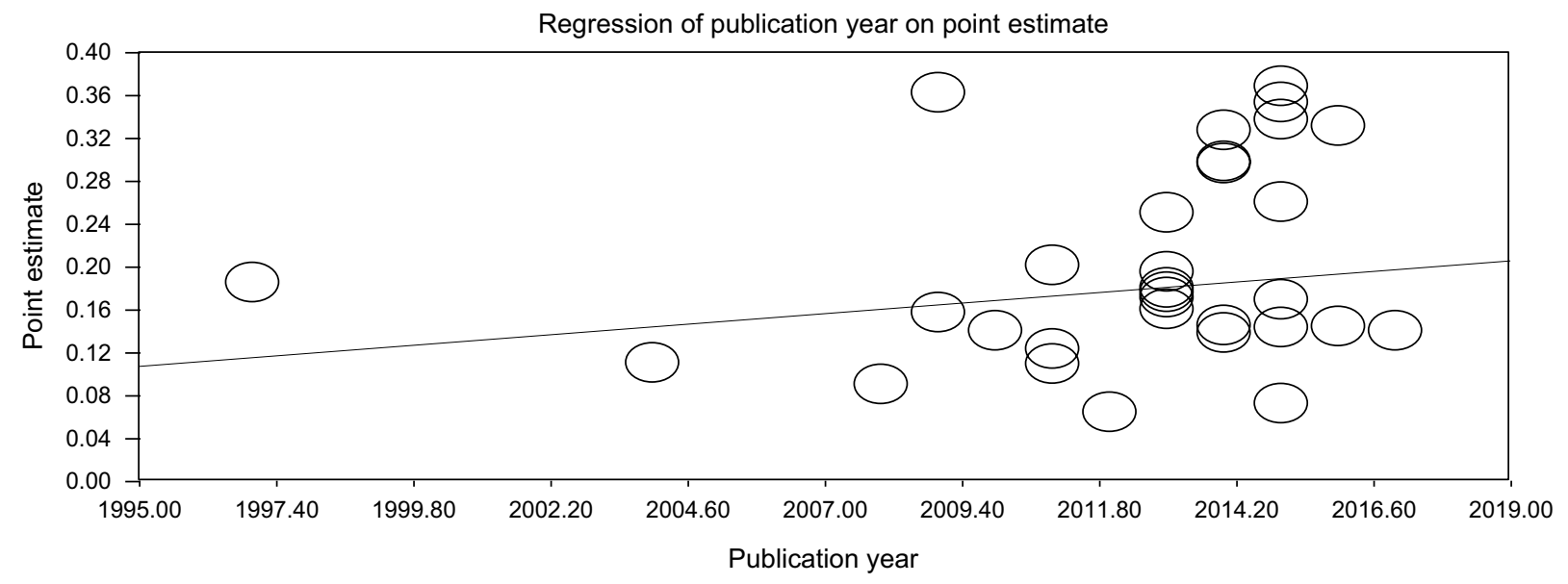

Figure 3 Scatter plot showing the effect of publication year on point estimate of prevalence of EPTB among PLWHA, SSA, 2017.

Abbreviations: EPTB, extrapulmonary tuberculosis; PLWHA, people living with HIVIAIDS; SSA, sub-Saharan Africa. 

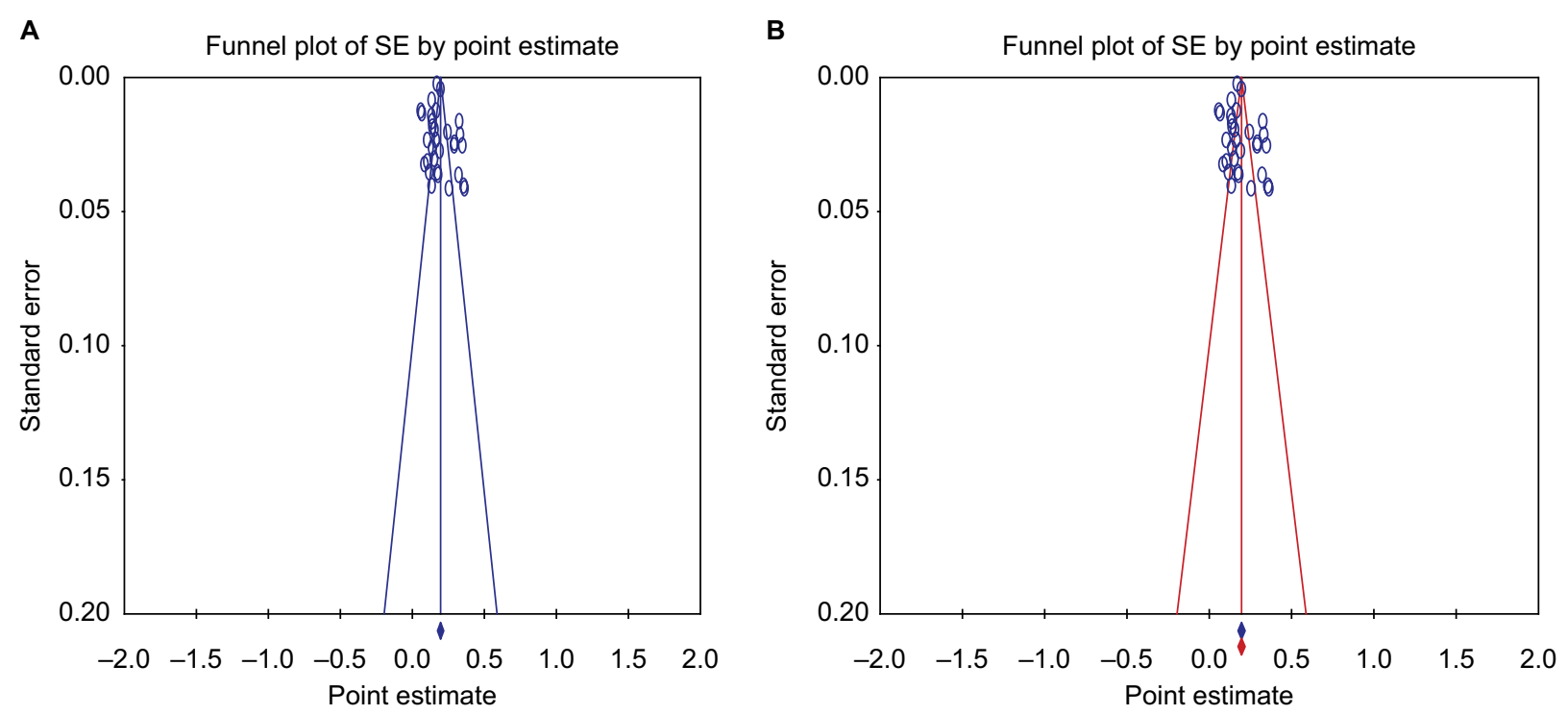

Figure 4 Funnel plot for SE by point estimate (A) for included studies (B) and input studies that shown similar pooled estimate. Abbreviation: SE, standard error.

\section{Discussion}

In our systematic review and meta-analysis of EPTB among PLWHA, we selected 31 studies with 28,659 HIV/AIDS people. Our main findings showed that there was a high and diverse prevalence of EPTB among PLWHA in SSA which ranged from $6.4 \%(95 \% \mathrm{CI}: 3.8,9.0)$ in Nigeria $^{29}$ to $36.8 \%$ (95\% CI: 28.6, 45.0) in Ethiopia. ${ }^{19}$ The overall pooled estimate prevalence of EPTB among PLWHA was 20\% (95\% CI: 17, 22; heterogeneity: $\tau^{2}=0 ; \chi^{2}=509.09, d f=30, P<0.00001$; $\left.I^{2}=94 \%\right)$.

This is high when we compared with the prevalence estimate of EPTB among notified TB cases in Africa. According to the WHO report of 2017, the prevalence of EPTB in Africa and world was $16 \%$ and $15 \%$, respectively, among notified TB cases in 2016. ${ }^{1}$ From the WHO report of 2017, we observed the prevalence of EPTB among notified TB cases for respective sub-Saharan countries, when we compared SSA by the geographical area, the eastern SSA was the lead, followed by Southern SSA, and the western was the least. One-third of the studies (ten of 31 studies) were conducted in Ethiopia, with highest prevalence of EPTB (32\%) from SSA in notified TB cases, followed by Malawi 26\%, Tanzania 21\%, Zambia $18 \%$, Burkina Faso, Kenya and Cameroon each 17\%, South Africa $10 \%$, Uganda $8 \%$, and Nigeria $5 \%{ }^{1}$

However, the pooled prevalence of EPTB among PLWHA in subregions of SSA showed a slight difference with highest from southern SSA, $21 \%$ (95\% CI: 15, 26); followed by eastern SSA, 20\% (95\% CI: 16, 23), and finally, western SSA, $19 \%$ (95\% CI: 13, 24). This might be explained that the prevalence of HIV in population was high for the countries with evidence that included in the synthesis. That is, in 2016, SSA had the highest HIV/AIDS burden by sharing $70 \%$ (25.5 million) of 36.7 million people living with HIV, $64 \%$ of 1.8 million newly infected, and $73 \%$ of 1.0 million deaths worldwide. ${ }^{2}$ The HIV/AIDS burden varies among subregions of SSA countries as eastern and southern Africa is the region hardest hit by HIV/AIDS with lion share, 53\% of 36.7 million PLWHA and western and central Africa shares $17 \%$, making $70 \%$ of PLWHA in SSA. ${ }^{2}$

In SSA, the pooled estimate of prevalence of EPTB among PLWHA by countries having two or more studies in subgroup analysis showed higher when compared with estimated prevalence of EPTB among notified TB cases for Africa, $16 \%$. For instance, in Cameroon from western Africa with three studies, the pooled prevalence was 25\% (95\% CI: 12,37$)$, which was more than national estimate $17 \%$, in Ethiopia from eastern with 10 studies was 23\% (95\% CI: 17,29$)$ that was less than national estimate of $32 \%$, and South Africa was $17 \%$ (95\% CI: 14, 20) more than national estimate $10 \%,{ }^{1}$ but comparable with study done in respective countries.

Although the clinical presentations of TB among PLWHA vary by stage of HIV-positive individuals, TB is the common disease among them. ${ }^{48}$ At an early stage of HIV infection, the ratio of PTB to EPTB is 80:20 and at late stage is 50:50. ${ }^{49}$ In SSA, TB is often the first manifestation of HIV infection, and it is the leading cause of death among HIV-infected individuals. ${ }^{50}$ Similarly, HIV infection is one of the most 
important risk factors associated with an increased risk of latent TB infection progressing to active TB disease. While only $10 \%$ of immunocompetent individuals infected with $M$. tuberculosis go on to develop active disease in their lifetime; $50 \%$ of those coinfected with HIV develop active TB. ${ }^{51}$

To reduce the burden of TB, the WHO's policy on collaborative TB/HIV activities recommends a combination of measures among HIV-infected individuals. These measures include intensified case finding, isoniazid preventive therapy, and infection control and antiretroviral therapy. ${ }^{7}$

Although good progress was made in countries of SSA for intensified TB case findings among HIV-positive individuals or vice versa, still more intensified case finding of TB among PLWHA shall be performed. More over, if TB screened among PLWHA, the documentation was low as $18 \%$ in Africa, thus, documentation needs more focus in Africa. ${ }^{1}$ In addition, the SSA that had reviewed studies had not fulfilled the three 90th objectives, which is to indicate that, the 1 st 90 means $90 \%$ of people who are living with HIV, know their HIV status(positive or negative), the 2nd 90 means $90 \%$ of people living with HIV are on ART treatment, and the 3rd 90 means among people using ART, 90\% of their virus is suppressed. ${ }^{2}$

These findings are potentially important from a public health perspective since it suggests that more attention should be paid to screen EPTB among PLWHA, especially in countries with high EPTB in population of SSA (eg, Ethiopia and Malawi) and high HIV prevalence in population (eg, southern African countries).

Our sensitivity analyses showed that our overall results had no difference when we excluded one study with largest sample size. ${ }^{32}$ The heterogeneity was substantial $\left(I^{2}=94 \%\right)$, and we performed subgroup analysis by age group, types of EPTB, study design, time period, subregion, and countries having two or more studies and observed reduced or zero heterogeneity (Table 3 ). Thus, we explored and investigated the sources of inconsistence and finally performed the pooled estimate. We determined the effect of publication year on effect estimate that also one of the sources of heterogeneity. We expected the clinical variation as EPTB could be varied based on the organ involved, and in the way that the setting at which laboratory investigation performed, by whom and by how many people performed and interpreted. In reviewed studies, there was study design variations. Some studies we reviewed were performed with retrospective review of medical records, this results in underestimating the prevalence of EPTB among PLWHA. Others could be conducted with cross-sectional design in which comes the difficulty to judge which comes first. ${ }^{52}$ Similarly, at the analysis stage, appropriate analysis was not conducted in some studies that is why we excluded some studies when the prevalence of EPTB was not calculated, or not clear, and/or no data.

Overall, the bias that could possibly occur in some of the individual studies included in our review was selection bias that resulted from primary exposure or outcome measurement as the diagnosis of EPTB is challenging. ${ }^{6}$ Especially, in HIV-positive individuals, TB is atypical, and some facilities where the studies conducted were at health centers or similar. In these facilities, the required material or profession might not present, and in some studies the HIV statuses of some EPTB patients were unknown.

Second, selection bias could occur at design stage, in which a majority of evidence suggested that 19 out of 31 studies were retrospective in nature. In this case, authors were endorsed to underway studies with entry criteria majorly including those records that had complete data and excluded incomplete that could lead to nonresponse or missed variables or cases even without indicating the proportion of incomplete records. ${ }^{53}$ Finally, the information bias particularly during data collection time could be committed in some studies included in the review as mentioned earlier, because more than half of the studies involved data abstraction from article-based register without piloting or standardizing data abstraction checklists in addition to poor data handling practice in low-income developing countries. ${ }^{54}$ Thus, in both cases, the prevalence of EPTB among PLWHA, we found in this review, could have been underestimated. For future study, we recommend that further details on exposures and outcome measurement, samples and sampling techniques, and analysis are required.

However, our study has derived its strengths from its capability of having estimating EPTB among PLWHA in double burdened and leading continent, Africa, although not without limitation. As there were diverse and overlapping diagnostic techniques that had been performed in studies we reviewed, we could not perform the analysis of diagnosis that limited us to compare the prevalence of EPTB among PLWHA by diagnostic techniques. In addition, we reviewed articles written and/or published only in English; data published in other languages were not obtained. We therefore recommend collaboration of researchers from different language backgrounds to do a more exhaustive research in the future. 


\section{Conclusion}

We can conclude from our analyses that the prevalence of EPTB among PLWHA was high. It is necessary to give emphasis for EPTB rather than assuming as a rare event and focusing only on PTB, specifically, when screening TB among PLWHA, widening the spectrum of screening to include all common EPTB types in countries with high EPTB and/or HIV prevalence in the population.

\section{Disclosure}

The authors report no conflicts of interest in this work.

\section{References}

1. World Health Organization. Global Tuberculosis Report. Geneva: World Health Organization; 2017. Licence: CC BY-NCSA 3.0 IGO. 2017.

2. UNAIDS. Joint United Nations Programme on HIV/AIDS (UNAIDS), Geneva: UNAIDS; 2017.

3. Ait-Khalid N, Alarcon E, Armengol R, et al. Management of Tuberculosis: A Guide to Essentials of Good Practice. 6th edn. Paris, France: International Union Ageinest Tuberculosis and Lung Disease; 2010.

4. World Health Organization. TB/HIV Clinical Manual. Geneva, Switzerland: World Health Organization; 2004.

5. Wani RS. Clinical manifestations of pulmonary and extra-pulmonary tuberculosis. South Sudan Med J. 2013;6(3):52-56.

6. World Health Organization. Diagnosis and Treatment of Smear-Negative Pulmonary and Extrapulmonary Tuberculosis Among Adults and Blescents. Geneva, Switzerland: World Health Organization; 2007.

7. World Health Organization. Collaborative TB/HIV Activities Guidelines for National Programmes and Other Stake Holders. Geneva, Switzerland: World Health Organization; 2012.

8. Dheda K, Ruhwald M, Theron G, Peter J, Yam WC. Point-of-care diagnosis of tuberculosis: Past, present and future. Asian Pacific Soc Respir. 2012;18:217-232.

9. World Health Organization. Global Tuberculosis Report. Geneva, Switzerland: World Health Organization; 2016.

10. Sinkala E, Gray S, Zulu I, et al. Clinical and ultrasonographic features of abdominal tuberculosis in HIV positive adults in Zambia. BMC Infect Dis. 2009;9:44-51.

11. Agbor AA, Bigna JJ, Billong SC, et al. Factors associated with death during tuberculosis treatment of patients co-infected with HIV at the Yaoundé Central Hospital, Cameroon: an 8-year hospital-based retrospective cohort study (2006-2013). PLoS One. 2014;9(12):e115211.

12. Nassikas N, Yang H, Forson A, Kwarteng E, Kwara A. Factors associated with mortality in extrapulmonary tuberculosis patients at a teaching hospital in Ghana. Ghana Med J. 2015;49(4):233-238.

13. Joanna Briggs Institute Reviewers' Manual. The Systematic Review of Prevalence and Incidence Data. South Australia: 2014.

14. Thorlund K, Imberger G, Johnston BC, et al. Evolution of heterogeneity (I2) estimates and their $95 \%$ confidence intervals in large meta-analyses. PLoS One. 2012;7(7):e39471.

15. Higgins JP, Thompson SG, Deeks JJ, Altman DG. Measuring inconsistency in meta-analyses. BMJ. 2003;327(7414):557-560.

16. Egger M, Davey Smith G, Schneider M, Minder C. Bias in meta-analysis detected by a simple, graphical test. BMJ. 1997;315(7109):629-634.

17. Moher D, Liberati A, Tetzlaff J, Altman DG, PRISMA Group. Preferred reporting items for systematic reviews and meta-analyses: the PRISMA statement. PLoS Med. 2009;6(7):e1000097-6.

18. Alemie GA, Gebreselassie F. Common types of tuberculosis and coinfection with HIV at private health institutions in Ethiopia: a cross sectional study. BMC Public Health. 2014;14:319-323.
19. Amare D. Tuberculosis and HIV co-infection among patients on tuberculosis treatment at Fenote Selam District Hospital, Amhara Regional State, Northwest Ethiopia. Global J Med Res. 2015;15(5):2249-4618.

20. Gebreegziabher SB, Yimer SA, Bjune GA. Tuberculosis case notification and treatment outcomes in West Gojjam Zone, Northwest Ethiopia: A Five-Year Retrospective Study. J Tuberc Res. 2016;4(1):23-33.

21. Deribew A, Negussu N, Melaku Z, Deribe K. Investigation outcomes of tuberculosis suspects in the health centers of Addis Ababa, Ethiopia. PLoS One. 2011;6(4):e18614.

22. Esmael A, Tsegaye G, Wubie M, Endris M. Tuberculosis and human immune deficiency virus co-infection in Debre Markos Referral Hospital in Northwest Ethiopia: a five years retrospective study. J AIDS Clin Res. 2013;4(12):263-267.

23. Hailu D, Abegaz WE, Belay M. Childhood tuberculosis and its treatment outcomes in Addis Ababa: a 5-years retrospective study. BMC Pediatr. 2014;14:61-67.

24. Mekonnen D, Awoke D, Endalkachew D. Tuberculosis and human immunodeficiency virus in western Burkina Faso: clinical presentation and clinical evaluation. BMC Res Notes. 2015;8:666-671.

25. Skogmar S, Balcha TT, Jemal ZH, et al. Development of a clinical scoring system for assessment of immunosuppression in patients with tuberculosis and HIV infection without access to CD4 cell testing-results from a cross-sectional study in Ethiopia. Glob Health Action. 2014;7:23105-23110.

26. Tarekegne D, Jemal M, Atanaw T, et al. Prevalence of human immunodeficiency virus infection in a cohort of tuberculosis patients at Metema Hospital, Northwest Ethiopia: a 3 years retrospective study. BMC Res Notes. 2016;9:192-197.

27. Yassin MA, Takele L, Gebresenbet S, et al. HIV and tuberculosis coinfection in the southern region of Ethiopia: a prospective epidemiological study. Scand J Infect Dis. 2004;36(9):670-673.

28. Daniel OJ, Adejumo OA, Gidado M, Abdur-Razzaq HA, Jaiyesimi EO. HIV-TB co-infection in children: associated factors and access to HIV services in Lagos, Niger. Public Health Action. 2015;5(3):165-169.

29. Affusim CC, Kesieme E, Abah VO. The Pattern of presentation and prevalence of tuberculosis in HIV-seropositive patients seen at Benin City, Nigeria. Int Scholar Res Network. 2012:1-6.

30. Adejumo OA, Daniel OJ, Otesanya AF, et al. Factors associated with TB/HIV co-infection among drug sensitive tuberculosis patients managed in a secondary health facility in Lagos, Nigeria. Afr J Infect Dis. 2017;11(2):75-82.

31. Iliyasu Z, Babashani M. Prevalence and predictors of tuberculosis coinfection among HIV-seropositive patients attending the Aminu Kano Teaching Hospital, northern Nigeria. J Epidemiol. 2009;19(2):81-87.

32. Gupta RK, Lawn SD, Bekker LG, Caldwell J, Kaplan R, Wood R. Impact of human immunodeficiency virus and CD4 count on tuberculosis diagnosis: analysis of city-wide data from Cape Town, South Africa. Int J Tuberc Lung Dis. 2013;17(8):1014-1022.

33. Hoffmann CJ, Hoffmann JD, Kensler C, et al. Tuberculosis and hepatic steatosis are prevalent liver pathology findings among HIV-infected patients in South Africa. PLoS One. 2015;10(2):e0117813.

34. Nglazi MD, Bekker LG, Wood R, Kaplan R. The impact of HIV status and antiretroviral treatment on TB treatment outcomes of new tuberculosis patients attending co-located TB and ART services in South Africa: a retrospective cohort study. BMC Infect Dis. 2015; 15:536-543

35. van der Plas H, Meintjes G, Schutz C, et al. Complications of antiretroviral therapy initiation in hospitalised patients with HIV-associated tuberculosis. PLoS One. 2013;8(2):e54145.

36. Namme LH, Solange DM, Bertrand MN, Elvis T, Ach JH, Christopher K. Extrapulmonary Tuberculosis and HIV co infection in patients treated for tuberculosis at the Doula Hospital in Cameroon. Ann Tropical Med Public Health. 2013;6(1):100-104.

37. Njozing NB, Miguel SS, Tih PM, Hurtig AK, Karin HA. Assessing the accessibility of HIV care packages among tuberculosis patients in the Northwest Region, Cameroon. BMC Public Health. 2010;10:129-139. 
38. Ocama P, Katwere M, Piloya T, et al. The spectrum of liver diseases in HIV infected individuals at an HIV treatment clinic in Kampala, Uganda. Afr Health Sci. 2008;8(1):8-12.

39. Wobudeya E, Lukoye D, Lubega IR, Mugabe F, Sekadde M, Musoke P. Epidemiology of tuberculosis in children in Kampala district, Uganda, 2009-2010; a retrospective cross-sectional study. BMC Public Health. 2015;15:967-1004.

40. Siddiqi OK, Ghebremichael M, Dang X, et al. Diagnosis of central nervous system opportunistic infections in HIV-infected Zambian adults. Clin Infect Dis. 2014;58(12):1771-1777.

41. Sinkala E, Gray S, Zulu I, et al. Clinical and ultrasonographic features of abdominal tuberculosis in HIV positive adults in Zambia. BMC Infect Dis. 2009;9:44-51.

42. Kamenju P, Said A. Tuberculosis-HIV co-infection among patients admitted at Muhimbili National Hospital in Dares salaam, Tanzania. Tanzania J Health Res. 2011;13(1):25-31.

43. Ade S, Harries AD, Trébucq A, et al. National profile and treatment outcomes of patients with extrapulmonary tuberculosis in Bénin. PLoS One. 2014;9(4):e95603.

44. Malkin JE, Prazuck T, Simonnet F, et al. Tuberculosis and human immunodeficiency virus in western Burkina Faso: clinical presentation and clinical evaluation. Int J Tubercul Lung Dis. 1999;1(1):68-74.

45. Feldacker C, Tweya H, Keiser O, et al. Characteristics of adults and children diagnosed with tuberculosis in Lilongwe, Malawi: findings from an integrated HIV/TB clinic. Trop Med Int Health. 2012;17(9):1108-1116.
46. Muttai H, Laserson KF, Akello I, et al. Antiretroviral therapy uptake among adult tuberculosis patients newly diagnosed with HIV in Nyanza Province, Kenya. Public Health Action. 2013;3(4):286-293.

47. Stolp SM, Huson MA, Janssen S, Beyeme JO, Grobusch MP. Tuberculosis patients hospitalized in the Albert Schweitzer Hospital, Lambaréné, Gabon-a retrospective observational study. Clin Microbiol Infect. 2013;19(11):E499-E501.

48. Leeds IL, Magee MJ, Kurbatova EV, et al. Site of extrapulmonary tuberculosis is associated with HIV infection. Clin Infect Dis. 2012;55(1):75-81.

49. Sharma SK, Mohan A, Kadhiravan T. HIV-TB co-infection: epidemiology, diagnosis \& management. Indian JMed Res. 2005;121(4):550-567.

50. Corbett EL, Marston B, Churchyard GJ, de Cock KM. Tuberculosis in sub-Saharan Africa: opportunities, challenges, and change in the era of antiretroviral treatment. Lancet. 2006;367(9514):926-937.

51. Adeiza M, Okpapi J, Abba A. HIV-Associated tuberculosis: A subSaharan African perspective. Sub-Saharan Afr J Med. 2014;1(1):1-14.

52. Song JW, Chung KC. Observational studies: cohort and case-control studies. Plast Reconstr Surg. 2010;126(6):2234-2242.

53. Benchimol EI, Smeeth L, Guttmann A, et al. The Reporting of studies Conducted using Observational Routinely-collected health Data (RECORD) statement. PLoS Med. 2015;12(10):e1001885.

54. Vassar M, Holzmann M. The retrospective chart review: important methodological considerations. J Educ Eval Health Prof. 2013;10:12-18.
HIV/AIDS - Research and Palliative Care

\section{Publish your work in this journal}

HIV/AIDS - Research and Palliative Care is an international, peerreviewed open access journal focusing on advances in research in HIV its clinical progression and management options including antiviral treatment, palliative care and public healthcare policies to control viral spread. The journal is included in PubMed. The manuscript man-

\section{Dovepress}

agement system is completely online and includes a very quick and fair peer-review system, which is all easy to use. Visit http://www.dovepress. com/testimonials.php to read real quotes from published authors. 